\title{
SUPPLEMENTARY INFORMATION SECTION
}

Chemicals: Dichloromethane, cyclohexane and methanol (Aldrich, fluorescence grade) were used without further purification and were subjected to blank experiments to ensure their fluorimetric purity.

All the standard PAH compounds used were commercially available (Aldrich) and were used without further purification. Three home-made PAH mixtures having different distributions of PAH were used. Their compositions are reported in Table 1.

The main differences among the mixtures were: the different concentration ratio of pyrene and fluorene (which is 1 in Mixture 1, 0.5 in Mixture 2, in Mixture 3 pyrene is absent), the absence in Mixture 2 and 3 of acenaphthylene, which is a species very abundant in flame, but not fluorescing, and the presence of other very fluorescing PAH present only in traces in flame, such as perylene and benzo(e)pyrene.

PAH Analysis : Polycyclic aromatic hydrocarbons from naphthalene (128 Da) up to coronene (300 Da), collectively called GC-PAH, were quantitatively analysed in the PAH-laden mixture collected in flame and in anthracene oil sample by gas chromatography-mass spectrometry (GCMS) on an HP5890 gas-chromatograph, equipped by a HP-5MS Crosslinked 5\% PhMe Siloxane $30 \mathrm{~m} * 0.25 \mathrm{~mm} * 0.25 \mu \mathrm{m}$ film thickness column, coupled with a HP5989A mass spectrometer equipped with an electron impact/chemical ionisation (EI/CI) ion source. The GC was programmed to operate from an initial temperature of $40^{\circ} \mathrm{C}(1 \mathrm{~min})$ to $300{ }^{\circ} \mathrm{C}$ at $10{ }^{\circ} \mathrm{C} / \mathrm{min}$. The injector temperature was $280{ }^{\circ} \mathrm{C}$ and the injector was operated in splitless mode. The quantification of PAH in the samples has been made on the basis of response factors evaluated analysing a standard mixture containing known concentrations of each PAH.

Samples: PAH-laden mixtures were isokinetically sampled by means of a sampling line constituted of a stainless-steel water-cooled probe $($ i.d. $=2 \mathrm{~mm})$, a condenser and a filter, in an atmospheric-pressure fuel-rich premixed $\mathrm{C}_{2} \mathrm{H}_{4} / \mathrm{O}_{2}$ flame $(\mathrm{C} / \mathrm{O}=0.8)$. The composition of a typical flame-formed sample is reported in Table 2. 


\section{TABLES}

Table 1 - Composition of standard PAH mixtures.

\begin{tabular}{|c|c|c|c|c|c|c|c|c|c|}
\hline \multirow[b]{2}{*}{ Compound } & \multirow{2}{*}{$\begin{array}{l}\text { Molecular } \\
\text { weight }\end{array}$} & \multicolumn{3}{|c|}{ Concentrations (mg/l) } & \multirow[b]{2}{*}{ Compound } & \multirow{2}{*}{$\begin{array}{c}\text { Molecular } \\
\text { weight }\end{array}$} & \multicolumn{3}{|c|}{ Concentrations (mg/l) } \\
\hline & & $\begin{array}{c}\text { Mixture } \\
1\end{array}$ & $\begin{array}{c}\text { Mixture } \\
2\end{array}$ & $\begin{array}{c}\text { Mixture } \\
3\end{array}$ & & & $\begin{array}{c}\text { Mixture } \\
1\end{array}$ & $\begin{array}{l}\text { Mixture } \\
2\end{array}$ & $\begin{array}{c}\text { Mixture } \\
3\end{array}$ \\
\hline Naphthalene & 128 & 17.2 & 2.68 & 29.1 & Benzo(a)anthracene & 226 & 10.2 & 2.68 & 7.55 \\
\hline Acenaphtylene & 152 & 17.2 & - & - & Chrysene & 228 & 8 & 2.68 & 11.5 \\
\hline Biphenyl & 154 & - & 2.68 & 8.7 & Benzo(b)fluoranthene & 252 & - & - & - \\
\hline Acenaphtene & 154 & - & 3.78 & 14.55 & Benzo(k)fluoranthene & 252 & - & - & - \\
\hline Fluorene & 166 & 8.6 & 5.37 & 10.55 & Benzo(e)pyrene & 252 & 8.6 & 3.78 & 6.5 \\
\hline Phenanthrene & 178 & 8.6 & 2.68 & 10.2 & Benzo(a)pyrene & 252 & 12 & 2.68 & 7.0 \\
\hline Anthracene & 178 & 17.2 & 2.68 & 10.5 & Perylene & 252 & 8.6 & 2.68 & 11.8 \\
\hline $\begin{array}{l}\text { 4H-Cyclopenta(def) } \\
\text { phenanthrene }\end{array}$ & 190 & - & 2.68 & 9.35 & $\begin{array}{l}\text { Indeno(1,2,3- } \\
\text { cde)pyrene }\end{array}$ & 276 & - & 3.78 & 8.65 \\
\hline Fluoranthene & 202 & 12 & 3.78 & 17.45 & Benzo(ghi)perylene & 276 & 9.8 & 2.68 & 22.25 \\
\hline Pyrene & 202 & - & 2.68 & - & Coronene & 300 & 8.6 & 3.78 & 3.6 \\
\hline Cyclopenta(cd)pyrene & 226 & 9.4 & 2.68 & 4.3 & Dibenzo(ai)pyrene & 302 & - & - & - \\
\hline
\end{tabular}


Table 2 - Composition of a PAH-laden mixture sampled in a fuel-rich premixed ethylene flame.

\begin{tabular}{cc}
\hline Compound & Concentration (\%) \\
\hline Naphthalene & 23.02 \\
Acenaphtylene & 46.58 \\
Biphenyl & 0.40 \\
Acenaphtene & 0.13 \\
Fluorene & 2.42 \\
Phenanthrene & 4.64 \\
Anthracene & 0.96 \\
4H-Cyclopenta(def) phenanthrene & 2.68 \\
Fluoranthene & 3.28 \\
Pyrene & 5.59 \\
Cyclopenta(cd)pyrene & 5.01 \\
Benzo(a)anthracene & 0.16 \\
Chrysene & 0.07 \\
Benzo(b)fluoranthene & 0.48 \\
Benzo(e)pyrene & 0.37 \\
Benzo(a)pyrene & 0.77 \\
Indeno(1,2,3-cde)pyrene & 0.58 \\
Benzo(ghi)perylene & 1.57 \\
Coronene & 1.26 \\
\hline & \\
\hline & \\
\hline & \\
\hline & \\
\hline &
\end{tabular}




\section{FIGURES}

Fig. 1- Measured and composite fluorescence emission spectra at fixed $\chi_{\text {exc }}=266 \mathrm{~nm}$ ) (a) and synchronous $(\Delta \lambda=20 \mathrm{~nm})$ (b) excitation wavelength of PAH Mixture 1 in dichloromethane solution.

Fig. 2 - Measured and composite fluorescence emission spectra at $\lambda_{\mathrm{exc}}=266 \mathrm{~nm}$ of anthracene oil in dichloromethane solution.

Fig. 3 - Comparison of fluorescence emission spectra $\lambda_{\text {exc }}=266 \mathrm{~nm}$ ) measured in cyclohexane solution and in dichloromethane solution of anthracene oil. 


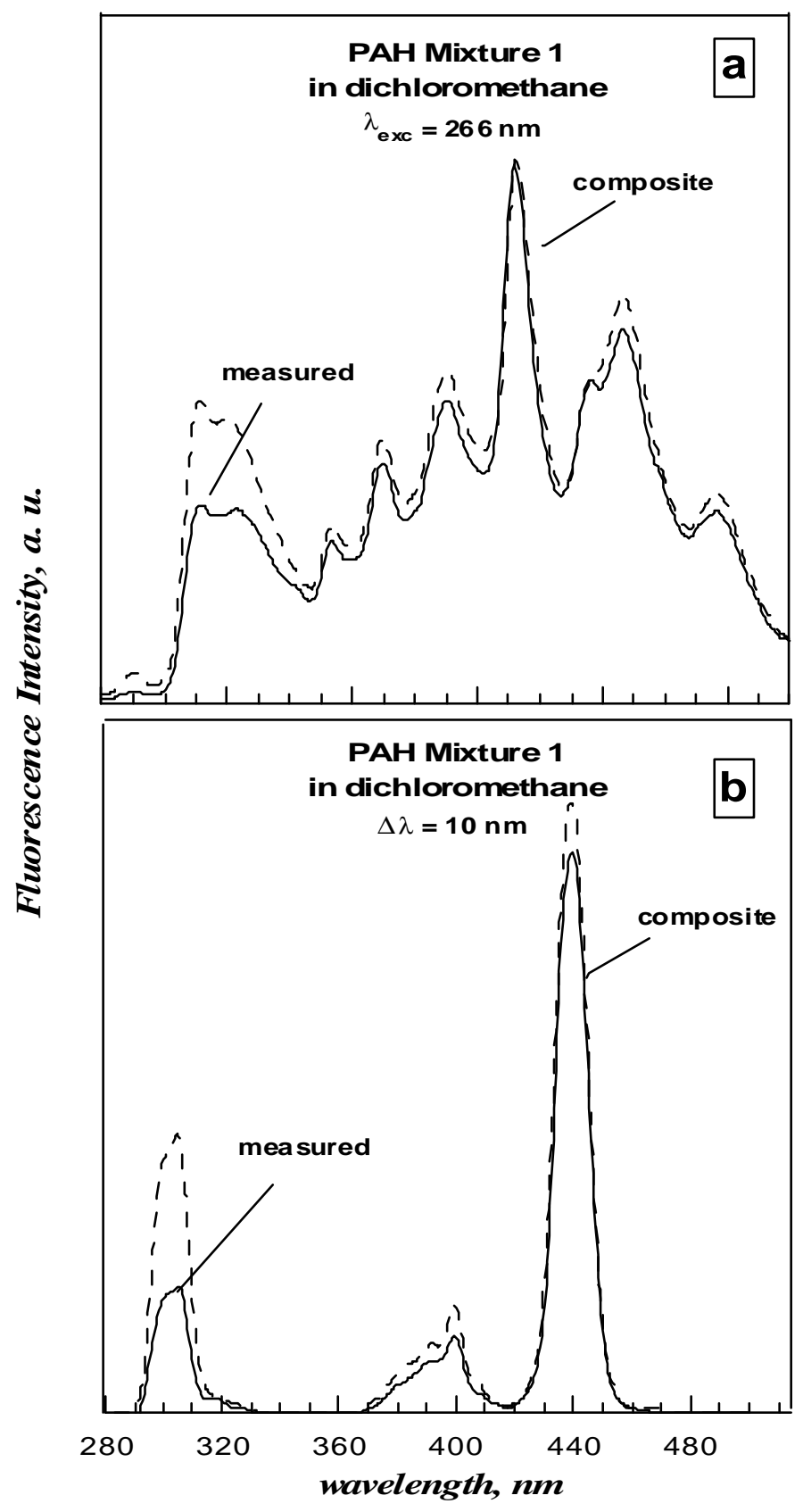

Fig.1 


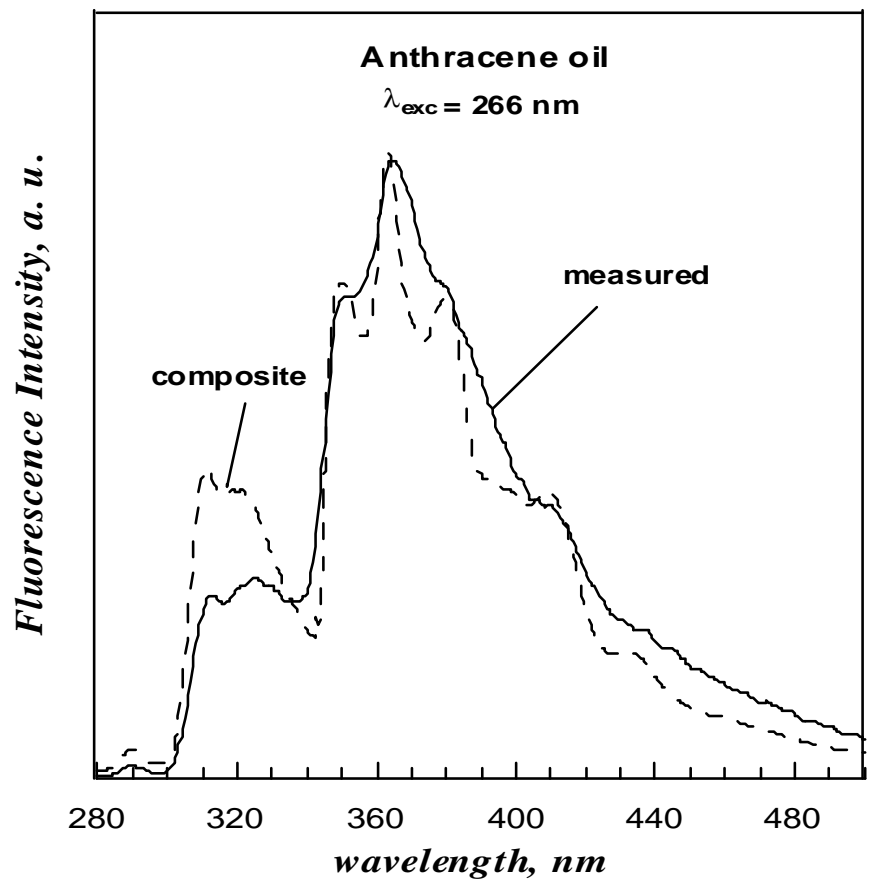

Fig.2 


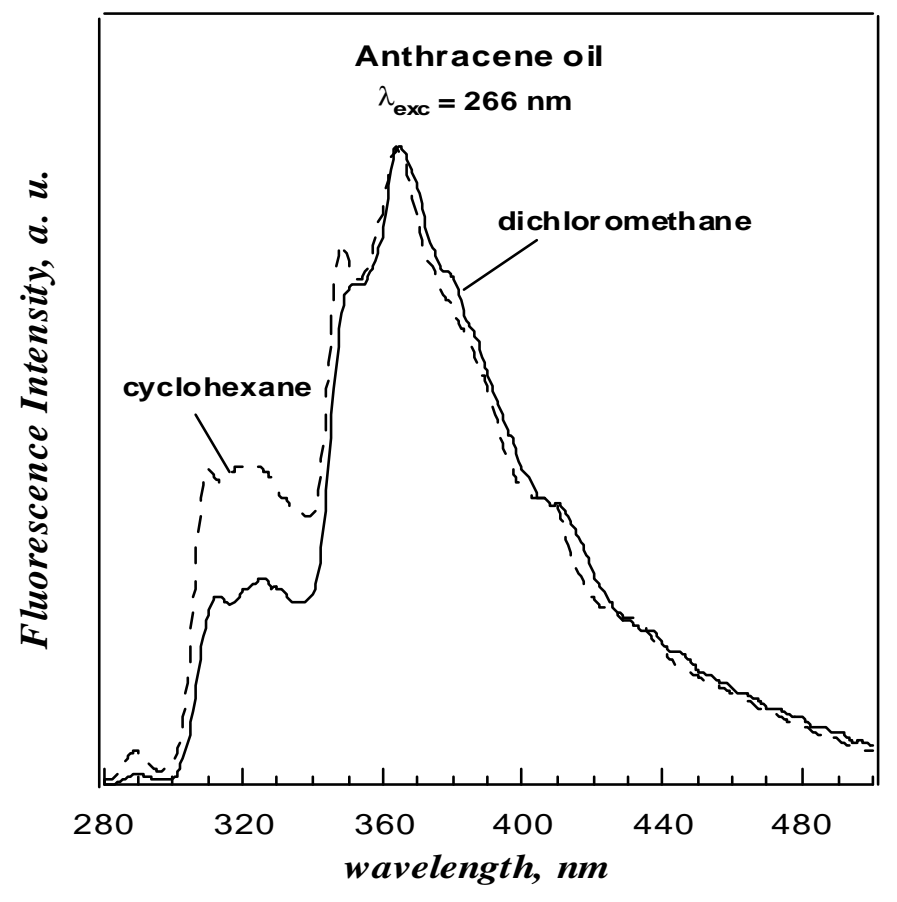

Fig.3 